\title{
Hand-Held Volatilome Analyzer Based on Elastically Deformable Nanofibers
}

\author{
Muge Yucel, ${ }^{\dagger}$ Osman Akin, ${ }^{\ddagger}$ Mehmet Cayoren, ${ }^{\S}$ Ibrahim Akduman, ${ }^{\S}$ Alagappan Palaniappan, \\ Bo Liedberg, ${ }^{\| \odot}$ Gurkan Hizal, $^{\perp}$ Fatih Inci, $^{\#}$ and Umit Hakan Yildiz, ${ }^{*}{ }^{\circ}$
}

${ }^{\dagger}$ Department of Biotechnology and Bioengineering, Izmir Institute of Technology, Izmir 35430, Turkey

${ }^{\ddagger}$ Department of Mechatronic Engineering, Izmir Katip Çelebi University, Izmir 35640, Turkey

${ }^{\S}$ Department of Electronic and Communication, Istanbul Technical University, Istanbul 34398, Turkey

"Center for Biomimetic Sensor Science, School of Materials Science and Engineering, Nanyang Technological University, 637553

Singapore

${ }^{\perp}$ Department of Chemistry, Istanbul Technical University, Istanbul 34398, Turkey

\# Department of Radiology, Stanford University, School of Medicine, Canary Center at Stanford for Cancer Early Detection, Palo Alto, California 94304, United States

ODepartment of Chemistry, Izmir Institute of Technology, Izmir 35430, Turkey

\section{Supporting Information}

ABSTRACT: This study reports on a hand-held volatilome analyzer for selective determination of clinically relevant biomarkers in exhaled breath. The sensing platform is based on electrospun polymer nanofiber-multiwalled carbon nanotube (MWCNT) sensing microchannels. Polymer nanofibers of poly(vinylidene fluoride) (PVDF), polystyrene (PS), and poly(methyl methacrylate) (PMMA) incorporated with MWCNT exhibits a stable response to interferences of humidity and $\mathrm{CO}_{2}$ and provides selective deformations upon exposure of exhaled breath target volatilomes acetone and toluene, exhibiting correlation to diabetes and lung cancer, respectively. The sensing microchannels "P1" (PVDF-MWCNT), "P2" (PS-MWCNT), and "P3" (PMMAMWCNT) are integrated with a microfluidic cartridge ( $\mu$-card) that facilitates collection and concentration of exhaled breath. The volatilome analyzer consists of a conductivity monitoring unit, signal conditioning circuitries and a low energy display module. A combinatorial operation algorithm was developed for analyzing normalized resistivity changes of the sensing microchannels upon exposure to breath in the concentration ranges between $35 \mathrm{ppb}$ and $3.0 \mathrm{ppm}$ for acetone and $1 \mathrm{ppb}$ and $10 \mathrm{ppm}$ for toluene. Subsequently, responses of volatilomes from individuals in the different risk groups of diabetes were evaluated for validation of the proposed methodology. We foresee that proposed methodology provides an avenue for rapid detection of volatilomes thereby enabling point of care diagnosis in high-risk group individuals.
$\mathrm{T}$ he need for low-cost, lightweight, and high performance sensing devices is continuously increasing because of the growing demand for miniaturized and portable instrumentation. Integrated biochips, microfluidic and sample enrichment devices have attracted significant interest for portable diagnostics. Noninvasive devices such as volatilome analyzers are becoming common practice for early biomarker detection and for continuous monitoring of disease development and treatment. Breath analysis for diagnosis offers numerous advantages, including rapid response time, simple sample collection, and pretreatment, as compared to other matrices such as saliva, feces, urine, blood, and sweat analyses. ${ }^{1-8}$ Moreover, recent efforts on identification metabolomics and

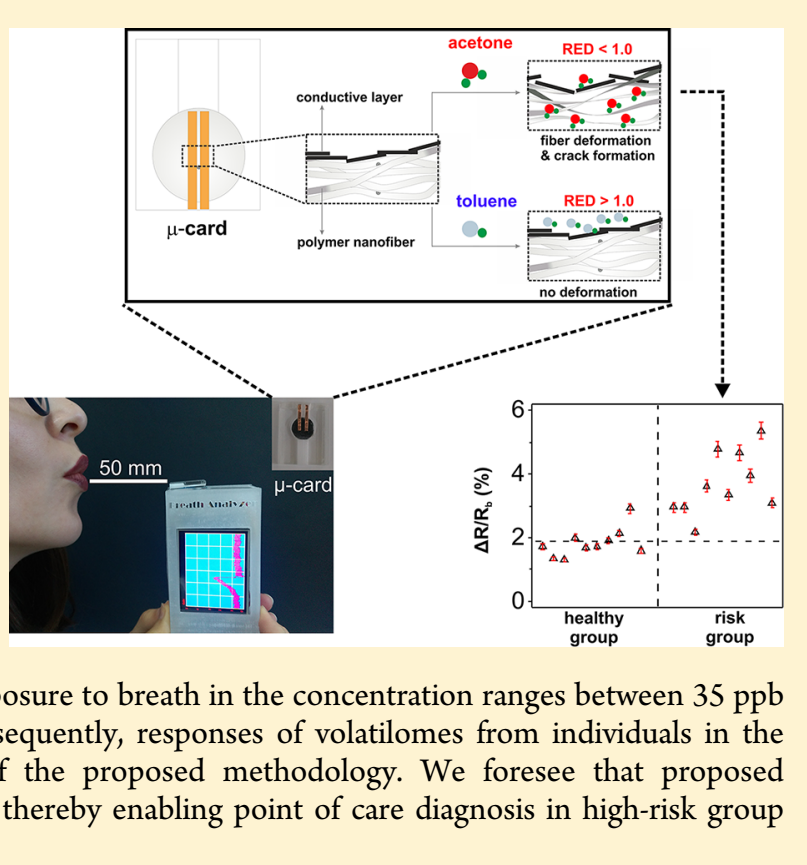

separation of 874 volatile organic compounds (VOCs) in exhaled breath expedites utilization of VOCs as potential biomarkers that correlate to various diseases. ${ }^{9-11}$ The impact of research in metabolomics speeds up collective advancements in preventive healthcare, drug toxicity studies, and disease modeling biomarkers discovery, thereby gaining importance in genomics and proteomics. Moreover, research on the metabolomic fingerprinting provides rapid screening of

Received: December 13, 2017

Accepted: March 20, 2018

Published: March 20, 2018 
Scheme 1. Operation and Components of Handheld Analyzer Consisting of an Integrated $\mu$-Card with Electrospun Polymer MWCNT Microchannels Attached to a Conductivity Monitoring System and a Display Unit (See Figure S2 for Circuit Board Details)

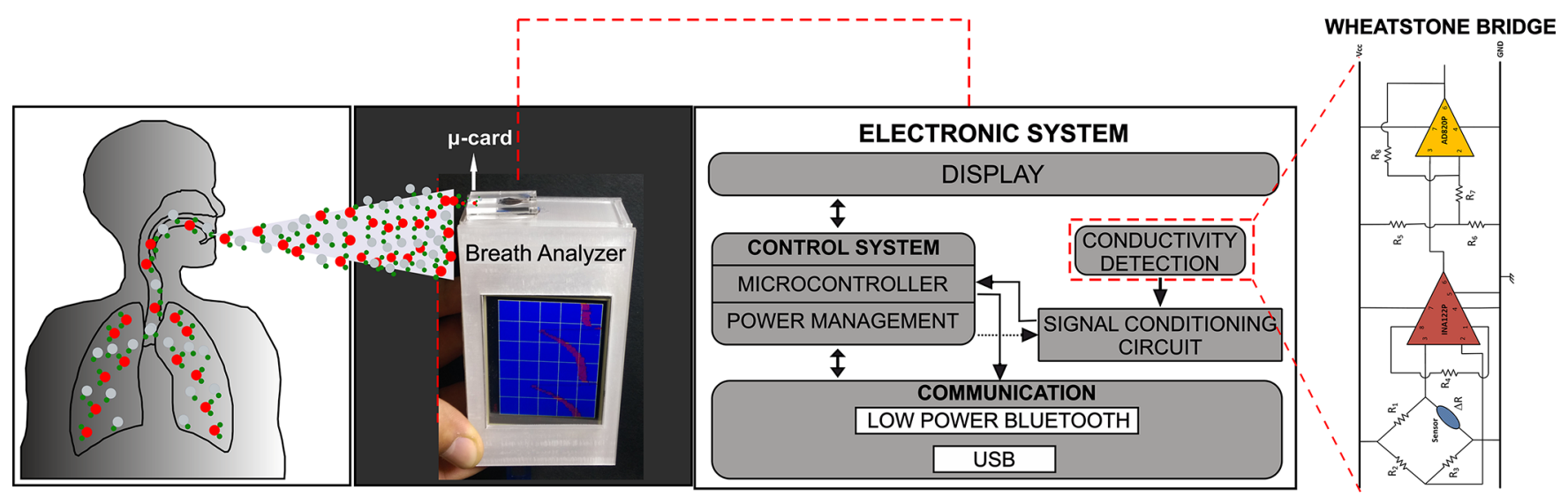

physiological conditions, enabling development of novel diagnostic approaches. ${ }^{12,13}$ Ongoing studies on diabetes, ${ }^{10,14-16}$ asthma, ${ }^{17}$ lung cancer, ${ }^{18-21}$ chronic obstructive pulmonary disease (COPD), ${ }^{22}$ and breast cancer are being diagnosed by screening of acetone, ${ }^{23,24} \mathrm{NO},{ }^{25,26}$ toluene, ${ }^{27-29}$ and $\mathrm{CO}$ in exhaled breath, respectively. ${ }^{30-34}$ Although VOCs are exhaled due to high volatility, ${ }^{35}$ their concentrations in breath are still very low (trace amount). Therefore, their detection often requires sophisticated instruments such as gas chromatography (GC), selected ion flow tube mass spectrometry (SIFT-MS), and proton transfer reaction mass spectrometry (PTR-MS). ${ }^{36}$ However, recent advances in microfabrication and nanotechnology have enabled fabrication of point of care biosensors that does not require bulky and sophisticated instrumentation. $^{28,37,38}$

During the past decade, numerous nanomaterials have been investigated in detail for VOC detection. Materials with nanoscale dimensions, for example, nanoparticles of various composition and size and carbon nanotubes (CNTs) offer significant advantages such as large surface-to-volume ratio and unique chemical, physical, and electrical properties. ${ }^{39}$ The large active surface area of these materials makes them extremely sensitive, as well as highly responsive. ${ }^{10}$ For instance, Chen et al. proposed a microfabricated breath sensor consisting of a pair of metal electrodes coated with a MWCNT layer with high detection sensitivity. ${ }^{40}$ Qi et al. prepared cellulose-CNT composite aerogel and recorded the resistivity changes in response to organic vapors with a simple, sensitive and stable methodology. ${ }^{41}$ Wang fabricated a hybrid structure of vertically aligned CNT-PEDOT-PS in which PEDOT enabled detection of analyte gases at low concentration and PS improved the selectivity. ${ }^{42}$ Multiwall carbon nanotube/polyimide (MWCNT/PI) composite film has been utilized for humidity sensing, where MWCNT was used to improve the sensitivity and linearity of the pristine PI sensor. ${ }^{43}$ Kybert et al. employed a DNA-CNT sensor to differentiate volatile organic compounds. ${ }^{44}$ These examples emphasize the potential of CNTbased VOC sensors in exhaled breath diagnostics. Moon et al. fabricated a volatilome analyzer based on Villi-like $\mathrm{WO}_{3}$ nanostructure to detect NO from exhaled breath. Inspired by intestinal Villi they deposited $\mathrm{WO}_{3}$ at glancing angles to increase the surface area of chemiresistor. The sensor could selectively detect $\mathrm{NO}$ with a limit of detection of $\sim 88 \mathrm{ppt}$ in a mixture of ethanol, acetone, $\mathrm{NH}_{3}$, and $\mathrm{CO}$, making it a potential platform for asthma diagnostics. ${ }^{45}$ In a follow-up study, a highly sensitive metal nanoparticle-based chemiresistive electronic nose was reported with enhanced sensitivities of 534 ppt from $2.87 \mathrm{ppb}$ for $\mathrm{H}_{2} \mathrm{~S}, 4.45 \mathrm{ppb}$ from $42.29 \mathrm{ppb}$ for $\mathrm{NH}_{3}$, and $206 \mathrm{ppt}$ from $2.06 \mathrm{ppb}$ for NO. ${ }^{28}$ Jung et al. developed a carbon-based volatilome analyzer for $\mathrm{NH}_{3}$ and $\mathrm{H}_{2} \mathrm{~S}$ sensor consisting of a single-stranded DNA (ssDNA)-functionalized graphene layer on a silicon substrate. They demonstrated sensitive detection of $\mathrm{NH}_{3}$ and $\mathrm{H}_{2} \mathrm{~S}$ (biomarkers for kidney disorders) in a highly humid environment. ${ }^{37}$ Kahn et al. designed an array of metal nanoparticle-based flexible sensor to detect different volatile organic compounds (VOCs) related to ovarian cancer. Each sensor element of the array was functionalized with ligands having unique functional groups. ${ }^{38}$ Ongoing follow up studies focus on strategies to enhance sensitivity and selectivity and to translate the platform into a diagnostic tool for clinical use. However, diminishing selectivity of the sensors because of the complex medium of real breath, deterioration of signal quality, and the broad variances in volume/composition of exhaled breath are still significant challenges to overcome, limiting the potential use of volatilome analyzers for disease diagnosis.

Herein we report on a hand-held volatilome analyzer for selective determination of clinically relevant biomarkers acetone and toluene (metabolic pathways of acetone and toluene given in SI section in detail). The sensing platform is based on electrospun polymer nanofiber-multiwalled carbon nanotube (MWCNT) sensing microchannel. Polymer nanofibers of poly(vinylidene fluoride) (PVDF), polystyrene (PS), and poly(methyl methacrylate) (PMMA) incorporated with MWCNT exhibit high tolerance to interfering humidity and $\mathrm{CO}_{2}$. The three polymer nanofibers were chosen because of their varying solubility properties (see Tables S1 and S2) in acetone and toluene, which are target volatilomes associated with diabetes and lung cancer. The major advantage provided by the PVDF nanofiber is the acetone specific deformation in the presence of interfering species that may exist in the exhaled breath. Similarly, PS nanofibers exhibit deformations upon exposure of toluene due to the matching of PS solubility parameter with toluene. The PMMA nanofibers utilized as control shows no specific deformations upon exposure of acetone and toluene. The proposed sensor configuration does not require sophisticated surface functionalization protocols exhibit negligible interferences, as long as the target's and 
Table 1. Electrospinning parameters of PVDF, PS, and PMMA

\begin{tabular}{lcccccc} 
& \multicolumn{2}{c}{ solution conditions } & & \multicolumn{3}{c}{ spinning conditions } \\
\cline { 2 - 3 } polymer & concn $($ wt $\%)$ & solvent composition by volume & & spinning distance $(\mathrm{mm})$ & voltage $(\mathrm{kV})$ & flow rate $(\mathrm{mL} / \mathrm{h})$ \\
PVDF & 25 & $\mathrm{DMF}(4) /$ acetone(1) & & 145 & 23 & 3.0 \\
PS & 15 & $\mathrm{DCM}(4) / \mathrm{DMF}(1) /$ acetone $(1)$ & & 120 & 25 & 3.5 \\
PMMA & 20 & $\mathrm{DMF}$ & 210 & 25 & 3.5 \\
\hline
\end{tabular}

nanofiber's solubility parameter matches. The microchannel sensing platform consists of a microfluidic cartridge ( $\mu$-card) that facilitates collection and preconcentration of exhaled breath. The $\mu$-card has a simplistic design and is integrated with an electronic interface that enables detection of minute resistivity changes over a large dynamic range. The volatilome analyzer with the $\mu$-card fitting socket holds great promise for screening of individuals in different risk groups. The detection methodology is briefly illustrated in Scheme 1. It enables rapid screening and extended monitoring of acetone in real breath. The described methodology provides excellent flexibility, sensitivity and specificity for future healthcare applications. The proposed methodology possesses a good potential for lowcost mass production of volatilome analyzers, enabling semicontinuous monitoring of VOCs in exhaled breath. Overall, the described noninvasive methodology enables rapid screening of volatilomes in breath and could be applicable for diagnosis of VOC related diseases.

\section{EXPERIMENTAL SECTION}

Materials. Poly(vinylidene fluoride) (PVDF; average molecular weight of $275.000 \mathrm{~g} / \mathrm{mol}$, Sigma-Aldrich), polystyrene (PS; average molecular weight of $280.000 \mathrm{~g} / \mathrm{mol}$, SigmaAldrich), and poly(methyl methacrylate) (PMMA; average molecular weight of $350.000 \mathrm{~g} / \mathrm{mol}$, Sigma-Aldrich) were utilized for fabrication of polymer nanofibers. Dimethylformamide (DMF, $\geq 99.8 \%$ ), dichloromethane (DCM, $\geq 99 \%$, SigmaAldrich), and acetone were used to dissolve polymers. Nitric acid $\left(\mathrm{HNO}_{3}, \geq 65 \%\right.$, Sigma-Aldrich), sulfuric acid $\left(\mathrm{H}_{2} \mathrm{SO}_{4}\right)$, hydrochloric acid ( $\mathrm{HCl}, \geq 37$, Sigma-Aldrich), and ammonium hydroxide $\left(\mathrm{NH}_{4} \mathrm{OH}, 26 \%\right.$, Sigma-Aldrich)) were used for the functionalization of MWCNTs. Acetone (ACS Rea. Merck) and toluene (ACS Rea. Merck) were used as volatilome vapors for 2-probe resistivity measurements.

Polymethyl methacrylate (PMMA) microchannel was assembled as a multilayer configuration that composed of four layers: (1) PMMA base, (2) nanofiber-CNT electrochemical membrane, (3) conductive metal tape, and (4) PMMA microfluidic channel. Sensing microchannel was designed to collect maximum amount of exhaled breath by means of a large opening in the front face that directs breath in to the sensing microchannels.

AFM Analysis. PVDF, PS, and PMMA were electrospun onto the gold surface that has been decontaminated in UV ozone cleaner for AFM analysis. Morphology analysis of single fiber was performed using tapping mode configuration with a scan size of $8.0 \mu \mathrm{m}$ and a scan rate of $1.0 \mathrm{~Hz}$. The characteristics of tip are length $=125 \mu \mathrm{m}$, width $=35 \mu \mathrm{m}$, frequency $=150 \mathrm{kHz}, k=5 \mathrm{~N} / \mathrm{m}$, and $r=8-12 \mathrm{~nm}$. The morphology of the fibers was systematically investigated prior and after solvent exposure. The fibers were purged with $\mathrm{N}_{2}$ for regeneration.

Electronic System. The electronic system consists of four main components: resistivity detection and signal conditioning circuit, microcontroller unit, communication unit that com- promise a USB, and a Bluetooth low energy module and display.

A highly sensitive Wheatstone bridge readout circuit was developed to convert the resistivity changes into voltage variations upon exhaled breath exposure to the sensing microchannels. The obtained signals were amplified to about 33-fold using an INA122 single supply instrumentation amplifier. Subsequently, the signal was amplified again using the AD820 opamp.

The Wheatstone Bridge (see Scheme 1) consists of four resistors; $R_{1}, R_{2}, R_{3}$, and the resistance of sensing microchannel defined as $R_{4}=R_{\text {base }}+\Delta R$, where $\Delta R$ is the resistivity variation upon exhaled breath exposure. In case there is no interaction between polymer nanofibers and volatilome in exhaled breath then, $R_{4}=R_{\text {base }}$, thereby the bridge will be in balance. However, upon any interaction between the analytes in exhaled breath with the polymer nanofibers, the resistance of $R_{4}$ changes $\left(R_{4}=\right.$ $R_{\text {base }}+\Delta R$ ), subsequently unbalancing the resistance bridge causing a voltage variation $V_{\text {bridge }}$ according to the equation:

$$
V_{\text {bridge }}=\left[\frac{R_{2}}{R_{1}+R_{2}}-\frac{R_{3}}{R_{3}+R_{4}}\right] \times
$$

where $V_{\mathrm{cc}}$ is the supply voltage set to $5 \mathrm{~V}$. Arduino Mega 2560 was utilized as the microcontroller unit. The analog voltage variations were processed by this microprocessor and the signals were transferred to the display for visualization. The portable device, see Scheme 1, is a hand-held device that is sensitive to resistivity changes. The hand-held device is composed of three components: $\mu$-card integrated circuit board, electronic biointerfacing circuit, and user communication interface with display and data transfer units. The validation experiments were performed by exhaled breath tests via monitoring the change in voltage variations over the time, evaluated from the resistivity changes of the sensing microchannels.

Normal Respiratory Flow Test. The volatilome analyzer was designed as portable hand-held diagnostic tool that has three functional units: LCD display, cartridge socket, and signal processing unit. The cartridge shown in Scheme 1 is made of sensing microchannels P1, P2, and P3 that were sandwiched between laser engraved top and bottom plastic substrates. Prior to volatilome detection, analyzer was first used to perform normal expiratory flow test to determine reference resistivity change upon breathing. In a typical analysis operation, analyzer was positioned at $50 \mathrm{~mm}$ from the mouth and exhaled breath was blown over the cartridge unit. For each analysis, breath is collected in a Tedlarbag from a healthy volunteer ( 23 years old, female).

\section{RESULTS AND DISCUSSION}

Characterization of Electrospun Polymer FiberMWCNT Microchannels. The sensing microchannels P1, P2, and P3 consist of polymer nanofibers that were fabricated by electrospinning. Table 1 shows the spinning parameters such 

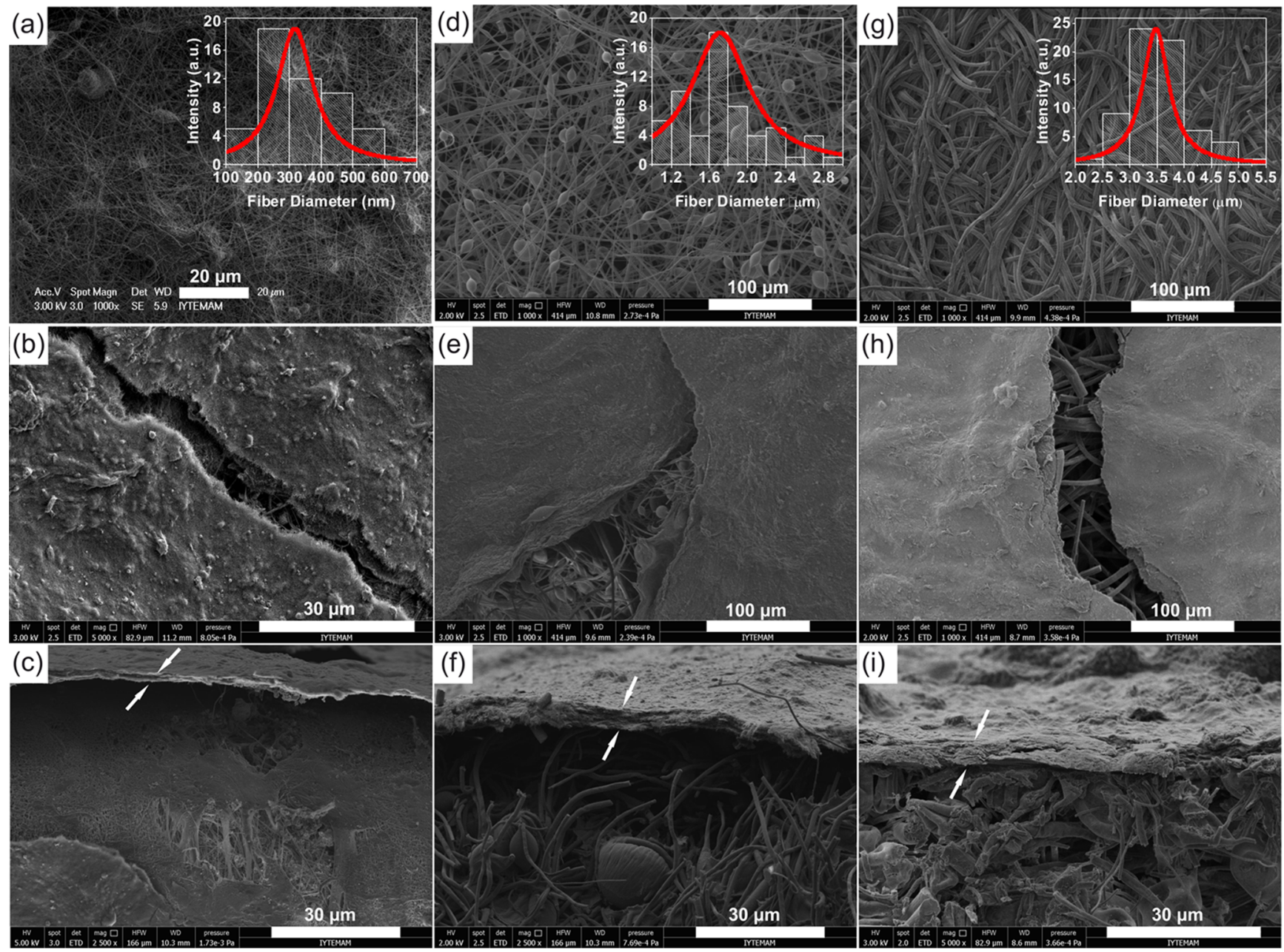

Figure 1. SEM images of polymer nanofibers: (a) PVDF nanofiber and PVDF-MWCNT, (b) top view and (c) side view; (d) PS nanofiber and PSMWCNT, (e) top view and (f) side view; (g) PMMA nanofiber and PMMA-MWCNT, (h) top view and (i) side view. Insets in a, d, and g show the distribution of fiber diameter.

as polymer concentration, solvent composition, spinning distance, voltage, and flow rate that have been optimized to control the morphology and the average diameter of the polymer nanofibers. The scanning electron micrographs of polymer nanofibers of PVDF, PS, and PMMA are shown in Figure 1.

Figure 1a, d, and g show that the average fiber diameter of the PVDF fibers are substantially smaller $(350( \pm 5.25) \mathrm{nm})$ than those of PS $(1.8( \pm 0.11) \mu \mathrm{m})$ and PMMA $(3.6( \pm 0.25)$ $\mu \mathrm{m})$, respectively, and the inset graphs illustrate the distribution of the fiber diameters. The PVDF and PMMA samples display a homogeneous distribution of fibers, whereas the PS fibers are less homogeneous, which is attributed to the formation of beads. The conductive MWCNT layer is generated on the polymer fiber mats by deposition from a $2 \mathrm{mg} / \mathrm{mL}$ stock solution with a conductivity of $29.4 \mu \mathrm{S} / \mathrm{cm}$. The conductive layer thicknesses are measured to be $3.5,8.4$, and $5.6 \mu \mathrm{m}$ for PVDF, PS, and PMMA, respectively (side view of sensing platforms are indicated by white arrows in Figure 1c,f,i). Cracks of varying sizes in the MWCNT coatings are seen for all PVDF, PMMA, and PS samples. Image analysis indicates that larger cracks are formed on PS and PMMA mats due to their higher average roughness and undulations with respect to those present on the PVDF mat. The crack sizes of MWCNT on PS and PMMA are approximately $5 \times$ larger than those on PVDF.
Moreover, the average base resistivity of the microchannels $\mathrm{P} 1$, $\mathrm{P} 2$, and $\mathrm{P} 3$ is $\sim 5 \mathrm{k} \Omega / \mathrm{cm}$.

Figure 2 shows AFM images of PVDF, PS, and PMMA fibers and the corresponding profile analyses in air and upon exposure to $1.5 \mathrm{ppm}$ of target volatilomes, acetone and toluene. The fiber diameters are $700 \mathrm{~nm}$ and 1.5 and $2.5 \mu \mathrm{m}$ for PVDF, PS, and PMMA, respectively (black curves). Significant deformations are seen upon acetone exposure. For instance, PVDF fibers exhibits changes in height and diameter of 14.3 and $9.9 \%$, respectively (see Figure 2d), whereas toluene introduces marginal changes in a single PVDF fiber. A substantial change in height $(36.3 \%)$ is evident for the PS fiber upon toluene exposure, contrary to acetone PMMA fibers that display very small changes in diameter and height upon exposure to acetone and toluene. The selective deformations of three polymer nanofibers upon acetone and toluene exposure are attributed to the relative permeability/solubility of gas molecules in polymer nanofibers. In order to gain a deeper understanding of the response of the polymers to the volatilomes, we considered the established theory relating the polymer solubility to the cohesive energy between polymer chains through Hildebrand solubility parameter $\delta=(-E / V)^{1 / 2}$, where $E$ is the cohesive energy (energy of vaporization) and $V$ is the molar volume of the pure solvent. ${ }^{46,47}$ The total cohesive energy, given by $E=$ $E_{\mathrm{D}}+E_{\mathrm{P}}+E_{\mathrm{H}}$, where $\mathrm{D}, \mathrm{P}$, and $\mathrm{H}$ refer to dispersion, dipole- 

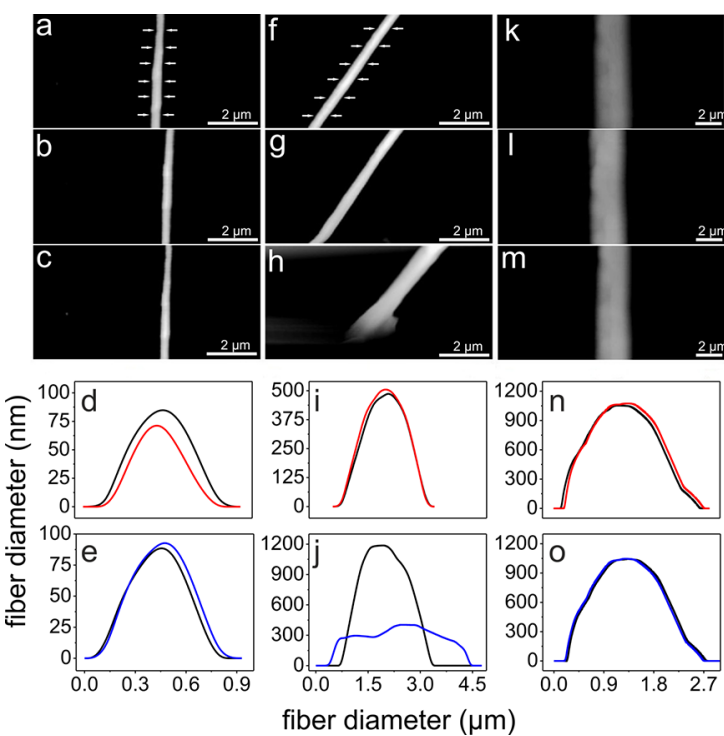

Figure 2. AFM images of polymer nanofibers: PVDF in (a) air, (b) acetone atmosphere, and (c) toluene atmosphere; PS in ( $\mathrm{f}$ ) air, (g) acetone atmosphere, and $(\mathrm{h})$ toluene atmosphere; PMMA in $(\mathrm{k})$ air, (l) acetone atmosphere, and $(\mathrm{m})$ toluene atmosphere. Prnalysis of PVDF in (d) air (black curve) and acetone atmosphere (red curve); (e) PVDF in air (black curve) and toluene atmosphere (blue curve); (i) PS in air (black curve) and acetone atmosphere (red curve); (j) PS in air (black curve) and toluene atmosphere (blue curve); (n) PMMA in air (black curve) and acetone atmosphere (red curve); (o) PMMA in air (black curve) and toluene atmosphere (blue curve).

dipole, and hydrogen bonding forces, respectively, is related to Hansen solubility parameter and the relative energy difference (RED) for the polymers (Table S3). ${ }^{48}$ In short, a volatilome molecule is expected to permeate into the polymer if the RED value is smaller than " 1 ", whereas no volatilome permeation occurs if the RED value is larger than " 1 ". ${ }^{49}$ The specific deformations seen in the PVDF and PS nanofiber networks upon acetone and toluene exposure correlate well to the RED values, thereby offering an avenue to differentiate target volatilomes in exhaled breath. Scheme 2 schematically illustrates the volatilome-induced nanofiber deformations that cause displacements and crack formation in the MWCNT conductive layer and a concomitant change in resistivity.

Scheme 2. Selective Response of the Sensing Microchannel Relies on the Extent of Volatilome Permeation into the Polymer Nanofiber Network ${ }^{a}$

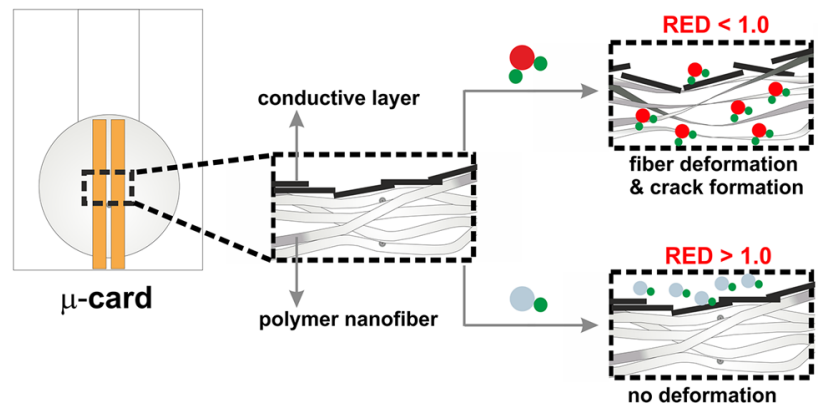

${ }^{a}$ RED values $<1$ induce deformation and crack formation in conductive layer, whereas RED values $>1$ do not allow for gas permeation and subsequent deformation and crack formation.
Sensing Performance and Fabrication of Volatilome Analyzer. Figure $3 \mathrm{a}$ briefly shows the fabrication steps $1-4$ of
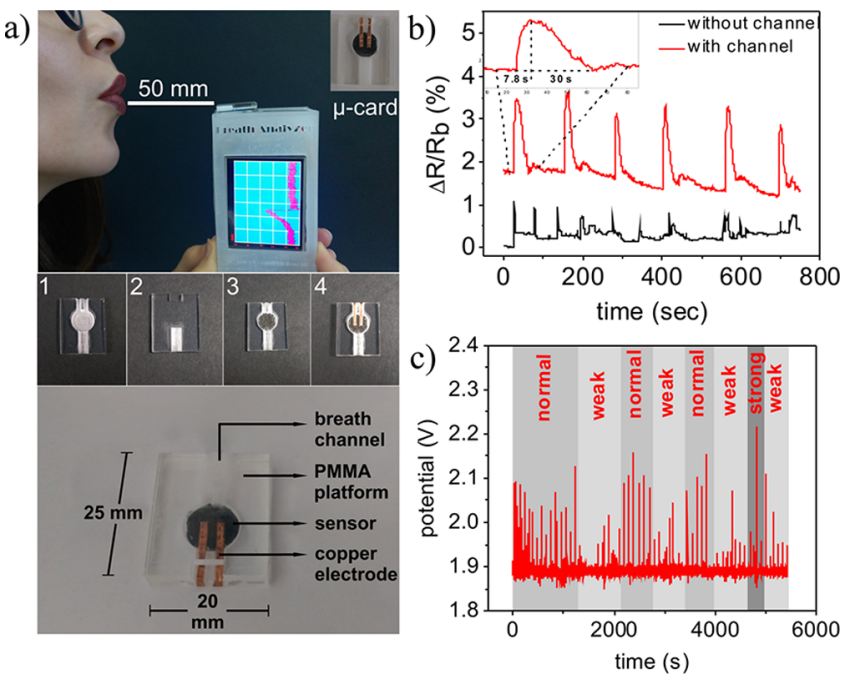

Figure 3. (a) Representation of volatilome analyzer and fabrication steps to obtain the integrated $\mu$-card: (1) bottom layer with sensing microchannel reservoir, (2) top layer, (3) sensing microchannel deposited on top bottom layer, (4) copper electrode deposition. (b) Typical resistivity graph of $\mu$-card with top sensing layer (red trace) and without sensing layer (black trace) for exhaled breath. (c) Typical potential graph of $\mu$-card for exhaled breath at different flow rates.

the sensing microchannel, the integrated $\mu$-card, and the volatilome analyzer. Figure $3 \mathrm{~b}$ shows the response to the collected breath flown over sensing microchannel (P1) at a rate of $6 \mathrm{~L} / \mathrm{min}$, yielding a reproducible relative response $\Delta R / R_{\mathrm{b}} \sim$ $1.5 \%$ (red trace). The magnified inset graph shows a response time for adsorption of $7.8 \mathrm{~s}$ and desorption of $30 \mathrm{~s}$. An identical test conducted using the nanofiber-MWCNT layer (black traces) without microchannel configuration yielded random and sharp fluctuations with no correlation to exhaled breath injection. This result indicates that the proposed configuration of a sensing microchannel with nanofiber-MWCNT coating yields a reproducible response profile (adsorption/desorption), facilitating exhaled breath monitoring. Responses to exhaled breath at varying flow rates categorized as weak $(3( \pm 0.15) \mathrm{L} /$ $\mathrm{min})$, normal $(6( \pm 0.3) \mathrm{L} / \mathrm{min})$, and strong $(10( \pm 0.5) \mathrm{L} / \mathrm{min})$, as shown in Figure $3 c$, yield a maximum voltage deviation of $1.95,2.15$, and $2.25 \mathrm{~V}$, respectively, illustrating a linear relation between the change in voltage $(\Delta V)$ and flow rate.

The volatilome detection capability of the analyzer with the $\mathrm{P} 1, \mathrm{P} 2$, and $\mathrm{P} 3$ sensing microchannels was evaluated by spiking acetone and toluene into breath samples collected from healthy donors. Figure $4 \mathrm{a}$ shows the acetone response for the three sensing microchannels for concentrations ranging between 0.3 and $3.2 \mathrm{ppm}$. The P1 sensing microchannel displays a linear response for concentrations between 0.3 and $1.7 \mathrm{ppm}$ (at a sensitivity of $24.4 \% / \mathrm{ppm}$ ). The P2 sensing microchannel displays a linear dependency throughout the entire concentrations regime $(0.3-3 \mathrm{ppm})$, but with a lower sensitivity $5.95 \% / \mathrm{ppm}$. The response for the P3 sensing microchannel is significantly lower with a sensitivity of $1.38 \% / \mathrm{ppm}$. Figure $4 \mathrm{~b}$ illustrates the responses of the three microchannels with P2 showing the highest sensitivity of $14.3 \%$ /ppm between $0.3-1.5$ ppm. P1 significantly deviates from linearity in the same concentration regime. As observed for acetone, P3 also displays 
a)

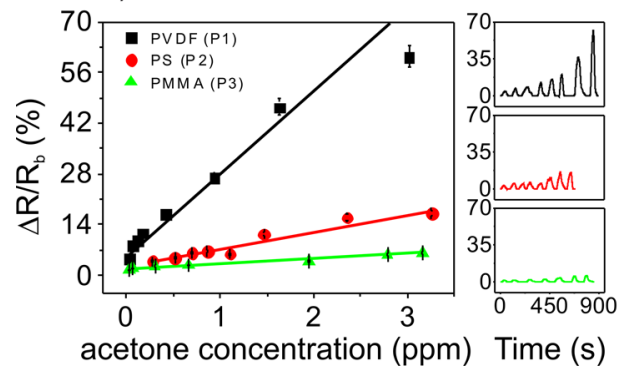

b)

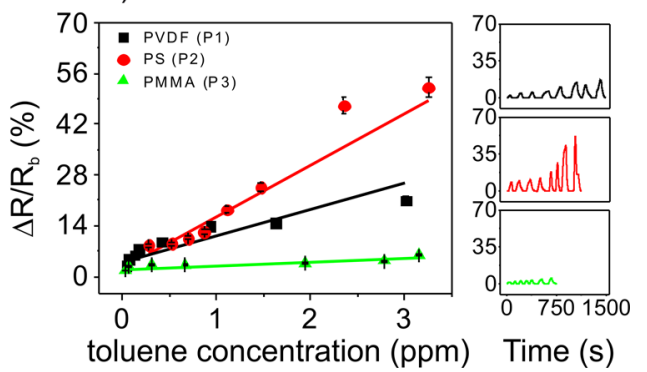

Figure 4. (a) Concentration-dependent acetone responses of PVDF (P1), PS (P2), and PMMA (P3). (b) Concentration dependent toluene responses of PVDF (P1), PS (P2), and PMMA (P3). The inset graphs in a and b show the concentration-dependent real-time responses of P1, P2, and $\mathrm{P} 3$ for acetone and toluene.

a low sensitivity to toluene. Figure 4a,b suggests that P1 and P2 yield selective responses to acetone and toluene. We also evaluated the signal reproducibility of the three microchannels by continuously exposing them to short acetone cycles (35 s) for $4000 \mathrm{~s}$, Figure 5a. The responses of the P1, P2, and P3

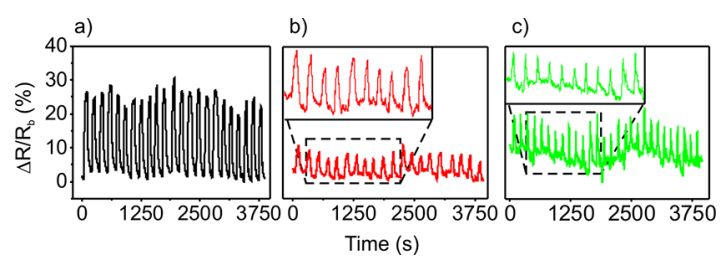

Figure 5. Signal reproducibility of (a) P1, (b) P2, and (c) P3 microchannels under sequential exposure to $0.9 \mathrm{ppm}$ acetone pulses (2 min) during $4000 \mathrm{~s}$.

microchannels are $24.02( \pm 2.2), 6.79( \pm 1.3)$, and 11.21 $( \pm 2.2)$, respectively. These responses illustrate that the three microchannels yield reliable and reproducible signals over an extended period of repetitive exposure to the volatilomes.

Volatilome Analysis in Exhaled Breath. A simple computational learning scheme was implemented by defining training sets based on concentration dependent $\Delta R / R_{\mathrm{b}}$ responses of target volatilomes. Here, the exhaled breath is considered as an input "I" for sensing microchannels P1, P2, and P3. Figure 6a shows $\Delta R / R_{\mathrm{b}}$ responses for increasing concentrations of volatilomes for P1, P2, and P3 microchannels. The variation in signal intensity is less than $2 \%$ for each sensing microchannel at $98 \%$ confidence level. Concentration-dependent responses of P1 for acetone and toluene are denoted as $\mathrm{I}_{1}$ and $\mathrm{I}_{1} *$, respectively. $\mathrm{I}_{1}$ and $\mathrm{I}_{1} *$ are normalized by factorizing the signals with reciprocal values of $\mathrm{I}_{1} . \mathrm{I}_{2}$ and $\mathrm{I}_{2}{ }^{*}$ are the response to acetone and toluene of $\mathrm{P} 2$ that were normalized by $\mathrm{I}_{2} *$ as described above. $\mathrm{I}_{3}$ and $\mathrm{I}_{3} *$ denotes the response of P3 to acetone and toluene, respectively. Figure $6 \mathrm{~b}$ shows the normalized response of sensing microchannels where $I_{1}, I_{2}^{*}$ yield a response of 1 and $\mathrm{I}_{1} *, \mathrm{I}_{2}$ yield a response between 0 and 1 that validates the truth table in Figure $6 \mathrm{c}$. The decision hardware and algorithm principle in Figure $6 \mathrm{c}$ responds to the inputs relying on the outputs summarized in "Decision Table", Figure $6 \mathrm{~d}$. Resistivity variations at the output of P1 and P2 are converted into voltage signals and applied to the OPAMP comparators (see Figure S2 for circuit boards design).

For $\mathrm{P} 1$, the normalized response $\mathrm{I}_{1}$ is connected to noninverting input of comparator, while $\mathrm{I}_{1} *$ is connected to the inverting input. If the input $\mathrm{I}$ is above the threshold value for acetone, then $\mathrm{I}_{1}$ is greater than $\mathrm{I}_{1} *$ and $V_{\text {out- } 1}$ is logic 1 . If it adopts a value below the threshold $V_{\text {out- } 1}$ is logic 0 . A similar logic is applied for P2. Accordingly, if input I is above the threshold value for toluene, then $\mathrm{I}_{2}{ }^{*}$ is greater than $\mathrm{I}_{2}$ and $V_{\text {out-2 }}$ is logic 1. The microchannel P3 is used for negative control of acetone and toluene. Thus, the presence of any components of these volatilomes in the breath will result in "LOW" level (logic

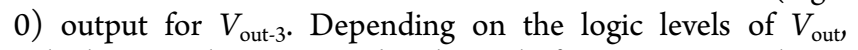
volatilome analyzer responds selectively for acetone or toluene in breath, for instance, if $V_{\text {out- } 1}=1, V_{\text {out-2 }}=0$, and $V_{\text {out-3 }}=0$ the analyzer utilizes the calibration curve in Figure $4 \mathrm{a}$ for detection of acetone concentration. If the output is $V_{\text {out- } 1}=0, V_{\text {out-2 }}=1$, and $V_{\text {out-3 }}=0$, the analyzer deduces toluene concentration based on calibration curve in Figure $4 \mathrm{~b}$. If the output of control sensing microchannel $V_{\text {out-3 }}=1$, analyzer deduces that neither acetone nor toluene exist in exhaled breath $\left(V_{\text {out-3 }}=1\right.$ was set to eliminate nonspecific response of interfering volatile organics). Upon validation of the proposed algorithm for recognition of target volatilomes using the functional training set, real breath sample from high-risk group individuals were used for proof of concept demonstration.

The exhaled breath screening of 20 individuals: healthy (10 volunteers) and risk group (10 volunteers, fasting blood sugar levels are between 100 and $125 \mathrm{mg} / \mathrm{dL}$, considered as prediabetic) were tested. In a typical operation, the volatilome analyzer was first set to autozero (P3 response) and then the test was initialized upon exhaling. As described previously, the presence of acetone will result in "HIGH" level (logic 1, yielding outputs, $\left.V_{\text {out-1 }}=1, V_{\text {out-2 }}=0, V_{\text {out- }-3}=0\right)$; subsequently, the concentration will be deduced based on calibration curve (Figure 4a). Figure 7 illustrates that the acetone response of healthy group is around $1.8 \Delta R / R_{\mathrm{b}}(\%)$, whereas the risk group's responses scatters between 2.9 and $5.3 \Delta R / R_{\mathrm{b}}$ (\%). The dotted line indicates the threshold acetone level in breath that corresponds to $10 \mathrm{ppm}$. All of the risk group individuals exhibit high levels of acetone with respect to threshold level, whereas the responses of healthy volunteers was observed to be either around or below the threshold level. The responses indicate that acetone concentration in exhaled breath of risk groups exhibit positive correlation and $t$ test was performed with unequal variances for the differentiation of acetone in exhaled breath of risk group individuals from the healthy group. The $t$ test yield a $t$ value of $5.36\left(t_{\text {stat }}>t_{\text {critical }}=2.16\right)$ corresponding to a statistically significant deviation as compared to the response of healthy group.

\section{CONCLUSIONS}

We have described a volatilome analyzer consisting of polymer nanofiber-multiwalled carbon nanotube composite sensing 

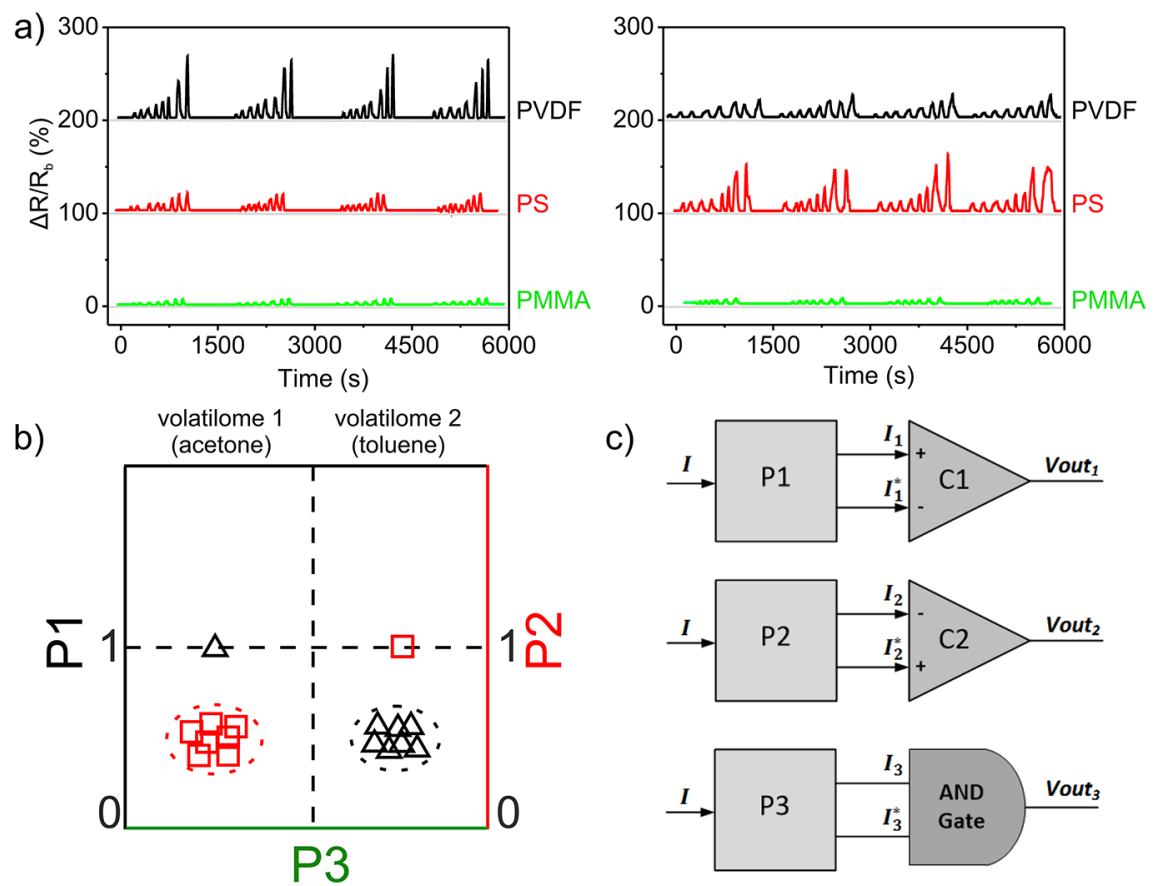

\begin{tabular}{|c|c|c|c|}
\hline$V_{\text {out }-1}$ & $V_{\text {out }-2}$ & $V_{\text {out }-3}$ & Result and Concentration Decision \\
\hline 1 & 0 & 0 & \multirow{3}{*}{$\begin{array}{l}\text { Decision for Input I: Acetone } \\
\text { Concentration: Calibration Curve for acetone in Figure 4a } \\
\text { Decision for Input I: Toluene } \\
\text { Concentration: Calibration Curve for toluene in Figure 4b } \\
\text { Decision for Input I: irrelevant }\end{array}$} \\
\hline 0 & 1 & $\mathbf{0}$ & \\
\hline 1 & $\mathbf{0}$ & 1 & \\
\hline 0 & 1 & 1 & Decision for Input l: irrelevant \\
\hline
\end{tabular}

Figure 6. (a) Volatilome responses of the sensing microchannels with increasing acetone concentrations (left) and toluene concentrations (right). (b) Normalized response of sensing microchannels P1, P2, and P3 upon input $\mathrm{I}_{1}, \mathrm{I}_{2} *$ and $\mathrm{I}_{1} *, \mathrm{I}_{2}$. (c) Hardware setup for volatilome analyzer (Cx: Comparator 1 and 2; P1, P2, and P3: sensing microchannels). (d) Decision table for volatilome recognition.

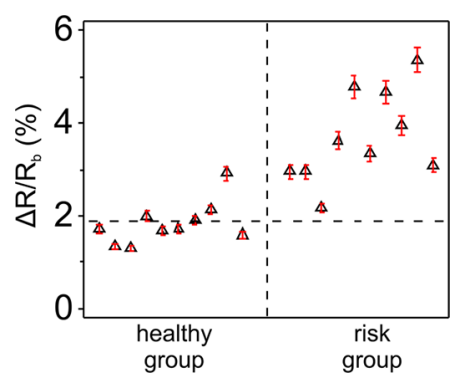

Figure 7. Typical normalized resistivity changes of P1 for breath of healthy and risk group individuals.

microchannel that respond selectively to acetone between the concentration range of $35 \mathrm{ppb}$ and $3.0 \mathrm{ppm}$ and toluene between $1 \mathrm{ppb}$ and $10 \mathrm{ppm}$ in exhaled breath. The suggested nanofiber based sensing platform enhances volatilome capture, thereby offering sensitive detection owing to the higher surfaceto-volume ratio of nanofibers. Moreover, these homogeneous nanofibers induce uniform CNT deposition across the sensing interface, which is critical for sensor performance as well as repeatability. The selective deformation of polymer nanofibers upon volatilome exposure is attributed to the relative permeability of gas molecules in polymer nanofiber. With a sensitivity of $24.4 \% / \mathrm{ppm}$ and a low deviation in $\% \Delta R / R_{\mathrm{b}}$, PVDF-MWCNT (P1) sensing microchannel enabled acetone detection from exhaled breath at clinically relevant concentration levels.

The volatilome analyzer and the proposed protocol for acetone screening possess the potential to facilitate prediabetic diagnosis of large group of individuals in a short time scale without blood sample collection. The correlation of proposed methodology with GC/Q-TOF-MS was found to be $98 \%$ in acetone detection (see Figure S4). Overall, the described methodology enables facile screening of acetone in exhaled breath by a-minute-long noninvasive test and could also be useful for diagnosis of VOC diseases.

\section{ASSOCIATED CONTENT}

\section{Supporting Information}

The Supporting Information is available free of charge on the ACS Publications website at DOI: 10.1021/acs.analchem.7b05187.

Experimental details, simulation results, and Hansen and Hildebrand and solubility parameter tables (PDF). 


\section{AUTHOR INFORMATION}

\section{Corresponding Author}

*E-mail: hakanyildiz@iyte.edu.tr; yildizuhakan@gmail.com. Fax: +90 2327507509 .

\section{ORCID $\odot$}

Muge Yucel: 0000-0001-7032-392X

Bo Liedberg: 0000-0003-2883-6953

Umit Hakan Yildiz: 0000-0002-6922-4454

Notes

The authors declare no competing financial interest.

\section{REFERENCES}

(1) Soini, H. A.; Klouckova, I.; Wiesler, D.; Oberzaucher, E.; Grammer, K.; Dixon, S. J.; Xu, Y.; Brereton, R. G.; Penn, D. J.; Novotny, M. V. J. Chem. Ecol. 2010, 36, 1035-1042.

(2) Garner, C. E.; Smith, S.; de Lacy Costello, B.; White, P.; Spencer, R.; Probert, C. S.; Ratcliffe, N. M. FASEB J. 2007, 21, 1675-1688.

(3) Khalid, T.; Aggio, R.; White, P.; De Lacy Costello, B.; Persad, R.; Al-Kateb, H.; Jones, P.; Probert, C. S.; Ratcliffe, N. PLoS One 2015, 10, e0143283.

(4) Pauling, L.; Robinson, A. B.; Teranishi, R.; Cary, P. Proc. Natl. Acad. Sci. U. S. A. 1971, 68, 2374-2376.

(5) Liu, C.; Furusawa, Y.; Hayashi, K. Sens. Actuators, B 2013, 183, $117-123$.

(6) Jadoon, S.; Karim, S.; Akram, M. R.; Kalsoom Khan, A.; Zia, M. A.; Siddiqi, A. R.; Murtaza, G. Int. J. Anal. Chem. 2015, 2015, 164974.

(7) Ulrike, T.; Hossam, H. J. Breath Res. 2014, 8, 027103.

(8) Kim, K. H.; Jahan, S. A.; Kabir, E. TrAC, Trends Anal. Chem. 2012, 33, 1-8.

(9) Amann, A.; Costello Bde, L.; Miekisch, W.; Schubert, J.; Buszewski, B.; Pleil, J.; Ratcliffe, N.; Risby, T. J. Breath Res. 2014, 8, 034001.

(10) Amann, A.; Miekisch, W.; Schubert, J.; Buszewski, B.; Ligor, T.; Jezierski, T.; Pleil, J.; Risby, T. Annu. Rev. Anal. Chem. 2014, 7, 455482.

(11) Poli, D.; Carbognani, P.; Corradi, M.; Goldoni, M.; Acampa, O.; Balbi, B.; Bianchi, L.; Rusca, M.; Mutti, A. Respir. Res. 2005, 6, 71.

(12) Lubes, G.; Goodarzi, M. J. Pharm. Biomed. Anal. 2018, 147, 318-322.

(13) Lubes, G.; Goodarzi, M. Chem. Rev. 2017, 117, 6399-6422.

(14) Kao, K. W.; Hsu, M. C.; Chang, Y. H.; Gwo, S.; Yeh, J. A. Sensors 2012, 12, 7157-7168.

(15) Minh, T. D. C.; Blake, D. R.; Galassetti, P. R. Diabetes Res. Clin.

Pract. 2012, 97, 195-205.

(16) Righettoni, M.; Tricoli, A. J. Breath Res. 2011, 5, 037109.

(17) Shorter, J. H.; Nelson, D. D.; McManus, J. B.; Zahniser, M. S.;

Sama, S. R.; Milton, D. K. J. Breath Res. 2011, 5, 037108.

(18) Dent, A. G.; Sutedja, T. G.; Zimmerman, P. V. J. Thorac Dis. 2013, 5 (Suppl 5), S540-S550.

(19) Chan, H. P.; Lewis, C.; Thomas, P. S. Lung Cancer 2009, 63, 164-168.

(20) Mazzone, P. J.; Hammel, J.; Dweik, R.; Na, J.; Czich, C.; Laskowski, D.; Mekhail, T. Thorax 2007, 62, 565-568.

(21) Taivans, I.; Bukovskis, M.; Strazda, G.; Jurka, N. Expert Rev. Anticancer Ther. 2014, 14, 121-123.

(22) Paredi, P.; Kharitonov, S. A.; Leak, D.; Ward, S.; Cramer, D.; Barnes, P. J. Am. J. Respir. Crit. Care Med. 2000, 162, 369-373.

(23) Qiao, Y.; Gao, Z.; Liu, Y.; Cheng, Y.; Yu, M.; Zhao, L.; Duan, Y.; Liu, Y. BioMed Res. Int. 2014, 2014, 869186.

(24) Choi, S. J.; Jang, B. H.; Lee, S. J.; Min, B. K.; Rothschild, A.; Kim, I. D. ACS Appl. Mater. Interfaces 2014, 6, 2588-2597.

(25) Yates, D. H. Immunol. Cell Biol. 2001, 79, 178-190.

(26) Pendharkar, S.; Mehta, S. Can. Respir. J. 2008, 15, 99-106.

(27) Capuano, R.; Santonico, M.; Pennazza, G.; Ghezzi, S.; Martinelli, E.; Roscioni, C.; Lucantoni, G.; Galluccio, G.; Paolesse, R.; Di Natale, C.; D’Amico, A. Sci. Rep. 2015, 5, 16491.
(28) Moon, H. G.; Jung, Y.; Han, S. D.; Shim, Y. S.; Shin, B.; Lee, T.; Kim, J. S.; Lee, S.; Jun, S. C.; Park, H. H.; Kim, C.; Kang, C. Y. ACS Appl. Mater. Interfaces 2016, 8, 20969-20976.

(29) Peralbo-Molina, A.; Calderon-Santiago, M.; Priego-Capote, F.; Jurado-Gamez, B.; Luque de Castro, M. D. J. Breath Res. 2016, 10, 026002 .

(30) Gardner, J. W.; Vincent, T. A. Sensors 2016, 16, 947.

(31) Ryter, S. W.; Choi, A. M. J. Breath Res. 2013, 7, 017111.

(32) Deveci, S. E.; Deveci, F.; Açik, Y.; Ozan, A. T. Respir. Med. 2004, 98, 551-556.

(33) Broza, Y. Y.; Haick, H. Nanomedicine 2013, 8, 785-806.

(34) Broza, Y. Y.; Mochalski, P.; Ruzsanyi, V.; Amann, A.; Haick, H. Angew. Chem., Int. Ed. 2015, 54, 11036-11048.

(35) Konvalina, G.; Haick, H. Acc. Chem. Res. 2014, 47, 66-76.

(36) Kim, I.-D.; Choi, S.-J.; Kim, S.-J.; Jang, J.-S. Exhaled Breath Sensors; Springer, 2015; pp 19-49.

(37) Jung, Y.; Moon, H. G.; Lim, C.; Choi, K.; Song, H. S.; Bae, S.; Kim, S. M.; Seo, M.; Lee, T.; Lee, S.; Park, H.-H.; Jun, S. C.; Kang, C.Y.; Kim, C. Adv. Funct. Mater. 2017, 27, 1700068.

(38) Kahn, N.; Lavie, O.; Paz, M.; Segev, Y.; Haick, H. Nano Lett. 2015, 15, 7023-7038.

(39) Nakhleh, M. K.; Amal, H.; Awad, H.; Gharra, A. 1.; Abu-Saleh, N.; Jeries, R.; Haick, H.; Abassi, Z. Nanomedicine 2014, 10, 17671776.

(40) Chen, X.; Wang, Y.; Wang, Y.; Hou, Z.; Xu, D.; Yang, Z.; Zhang, Y. Sens. Actuators, A 2010, 158, 328-334.

(41) Qi, H.; Liu, J.; Pionteck, J.; Pötschke, P.; Mäder, E. Sens. Actuators, B 2015, 213, 20-26.

(42) Wang, X.; Ugur, A.; Goktas, H.; Chen, N.; Wang, M.; Lachman, N.; Kalfon-Cohen, E.; Fang, W.; Wardle, B. L.; Gleason, K. K. ACS Sensors 2016, 1, 374-383.

(43) Yoo, K.-P.; Lim, L.-T.; Min, N.-K.; Lee, M. J.; Lee, C. J.; Park, C.-W. Sens. Actuators, B 2010, 145, 120-125.

(44) Kybert, N. J.; Lerner, M. B.; Yodh, J. S.; Preti, G.; Johnson, A. T. C. ACS Nano 2013, 7, 2800-2807.

(45) Moon, H. G.; Choi, Y. R.; Shim, Y. S.; Choi, K. I.; Lee, J. H.; Kim, J. S.; Yoon, S. J.; Park, H. H.; Kang, C. Y.; Jang, H. W. ACS Appl. Mater. Interfaces 2013, 5, 10591-10596.

(46) Rumens, C. V.; Ziai, M. A.; Belsey, K. E.; Batchelor, J. C.; Holder, S. J. J. Mater. Chem. C 2015, 3, 10091-10098.

(47) Lee, J. N.; Park, C.; Whitesides, G. M. Anal. Chem. 2003, 75, 6544-6554.

(48) Charles, M. H. Hansen Solubility Parameters: A User's Handbook, 2nd ed.; CRC Press, 2007.

(49) Salman, R. K.; Salih, J. M. IJMSA 2016, 5, 4858-4863. 